\title{
Identification of Dangerous Goods in Human THZ Images
}

\author{
Hong Xiao ${ }^{1, \text { a) }}$, Feng Zhu ${ }^{2, \text { b) }}$ \\ ${ }^{1}$ School of Computer Science, Guangdong University of Technology, China. \\ ${ }^{2}$ School of Information Engineering, Guangdong University of Technology, Guangzhou 510006, China \\ a) Zhu Feng: 302761359@qq.com \\ b) Xiao Hong:wh_red@163.com
}

\begin{abstract}
For terahertz image with low signal-to-noise ratio, serious blur and poor resolution, this paper uses the mean filter to denoise the terahertz image, and then uses Faster RCNN algorithm to detect and identify the dangerous goods in the terahertz image. Different from traditional algorithms, Faster RCNN algorithm uses traditional detection algorithms to locate, segment, extract effective features, integrate detection and recognition, and achieve automatic and rapid detection of hidden objects in the human body. The experimental results show that the proposed algorithm can effectively identify the dangerous articles of controlled knives in terahertz images, and the recognition rate can reach $89.6 \%$.
\end{abstract}

Key words: signal-to-noise; RCNN; automatic and rapid; recognition rate; terahertz.

\section{INTRODUCTION}

X-rays and other radiation have a certain degree of harm to the human body. At present, most security systems perform X-ray detection on items such as luggage, while terahertz waves are interposed between microwaves and infrared light, causing little harm to the substances to be detected and the human body, and radiation to clothes, Shoes, cartons and other materials have good penetrability and can be used to image items hidden under these materials and find hidden dangerous goods. Because of the low signal-to-noise ratio of terahertz image, serious blur, and poor resolution, most of the current terahertz imaging safety inspections rely on the human eye to identify, which can easily lead to human eye fatigue, resulting in false or missed detection, inefficient.

In response to the above problems, most studies currently focus on image denoising, enhancement, image segmentation, and detection. Generally, the image denoising enhancement of the terahertz image is improved by the algorithms such as gray scale transformation, Laplacian Gaussian operator, norm optimization constraint, and enhancement filtering to improve the image quality. The threshold segmentation algorithm is used to segment the terahertz image, and the terahertz image is detected by the LOG operator and fuzzy local information C-means clustering algorithm. However, the use of deep learning algorithms to identify terahertz images is still in its infancy.

This paper proposes the fusion of mean filter and deep learning algorithm Faster RCNN for the identification of dangerous goods in terahertz images. (1) The mean-value filtering of human terahertz images is used for decontamination. (2) Detection and recognition of controlled tools in human terahertz images using Faster RCNN. (3) Compare with other methods to analyze experimental results and performance.

\section{Related Work}

Because terahertz image has low signal-to-noise ratio, serious blur, and poor resolution, this makes it difficult to recognize and detect dangerous goods in terahertz images, and the recognition accuracy is not high. Currently, terahertz image processing research mainly adopts traditional image processing algorithms to improve terahertz image quality and image detection and recognition. 
In improving terahertz image quality, Zhu Weiwen et al. proposed an improved mean-filtering algorithm, adding the concept of threshold to the original mean-filtering algorithm, improving the image quality and increasing the ability to distinguish hidden objects. [1]. Sun Jianzhong et al. used the improved optimization of the threshold gray transformation algorithm to de-noise the terahertz wave image, and then sharpen the image based on the Laplacian operator to enhance the image quality [2].

In the aspect of image location detection, Feng Yanping and Wang Hui used threshold segmentation threshold to segment dangerous goods from the background, and then used LOG operator to extract edge features to achieve detection of gun THz images [3]. Jiang Tian et al. used the clustering algorithm based on fuzzy local information Cmeans for target detection and improved the membership function for the terahertz image properties [4].

From the above, we can see that the current research on terahertz image mainly lies in the improvement and detection of image quality. The adoption method is also the traditional image detection method. There is not much research available for the use of deep learning methods for the detection and identification of terahertz dangerous goods. The Faster RCNN [5] algorithm has been widely used in image recognition and classification since Ross Girsh's proposal in 2016. Faster RCNN detection speed and high accuracy, and the realization of the end-to-end target detection framework can be well utilized in the detection and identification of dangerous goods in terahertz. In view of the current security requirements, this paper proposes image enhancement based on exponential non-linear transformation and Faster RCNN algorithm to detect and identify suspicious objects belonging to controlled knives and other dangerous goods.

\section{THZ IMAGE PROCESSING}

\section{Mean Filtering Algorithm}

Since the terahertz wavelength is longer than the visible light and interferes with the imaging device and the external environment, the generated image is heavily blurred, the resolution is low, and the image quality is very poor. Before performing detection and identification, human body terahertz images are denoised to facilitate subsequent processing. We use the mean filter algorithm whose kernel function is:

$$
g(x, y)=\frac{1}{M} \sum f(x, y)
$$

Where $\mathrm{M}$ is the total number of pixels in the selected area, which $f(x, y)$ is the value filtered from around the point $(x, y)$, and $g(x, y)$ is the value generated after processing.

\section{Faster RCNN Algorithm}

Methods for detecting terahertz images Most of the above documents use traditional methods for detection and recognition. However, the method of deep learning has not been applied to the detection and recognition of terahertz images. Deep learning has been widely used in image detection and recognition. Therefore, this paper uses Faster $\mathrm{RCNN}$ to detect and recognize human terahertz maps.

After the accumulation of RCNN and Fast RCNN, Ross B. Girshick proposed a new Faster RCNN in 2015. In terms of structure, Faster RCNN has extracted feature extraction, proposal extraction, and bounding box regression. The classification is integrated in a network, which results in a significant increase in overall performance, especially in the detection speed.

Faster RCNN Framework 


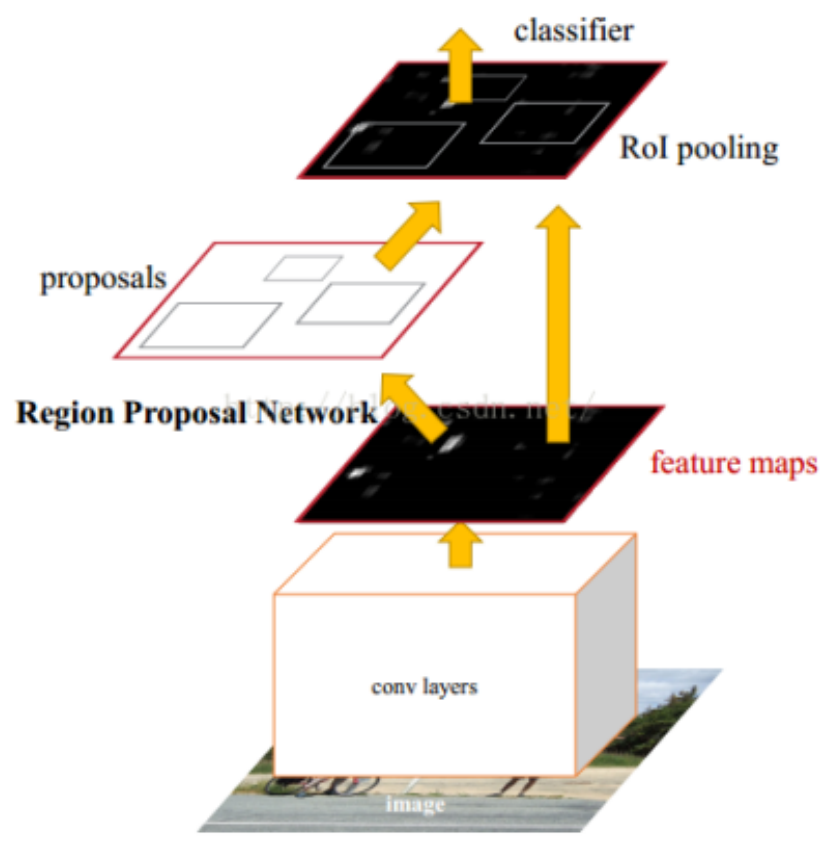

FIGURE 1. Faster R-CNN model

Faster RCNN can actually be divided into four main areas:

Conv layers: As a CNN network target detection method, Faster RCNN first uses a set of basic conv+relu+pooling layers to extract the feature maps of the image. $\mathrm{T}$

Region Proposal Networks: The RPN network is used to generate region proposals.

RoI Pooling: This layer collects the input feature maps and proposals.

Classification: Use proposal features maps to calculate the category of proposal, while again bounding box regression to get the final precise position of the detection frame.

This article uses the VGG16 model in the Python version.

The steps of Faster RCNN detection and identification of controlled tools in human THz images are:

1. Input human terahertz image training set image;

2.Input the picture into CNN for feature extraction;

3.Generate proposal windows using RPN, and generate 300 suggestion windows for each image;

4. Map the suggestion window to the last layer of the $\mathrm{CNN}$ feature map;

5.Generate a fixed-size feature map for each RoI through the RoI pooling layer;

6.Using the combination of Softmax Loss (detection classification probability) and Smooth L1 Loss (probe detection regression) for classification probability and Bounding box regression;

7.After the training, a model is generated, and then the test set image is put into the model for detection and identification.

\section{EXPERIMENTS AND ANALYSIS}

The verification experiments of this algorithm are all completed in the same system environment. The image processing experiment platform is a process of installing a 64-bit Win 10 system, the CPU is $17-6700 \mathrm{~K} * 8$, the main frequency is $4.0 \mathrm{GHz}$, and the memory is $16 \mathrm{~GB}$. The GPU is GTX750Ti + GTX1080. Terahertz images are processed using OpenCV-2.4.11+Python-2.7+ Caffe+Faster RCNN. The experimental image is a 472 (400 pixel $\times 160$ pixel) test set human terahertz image, and a 107 (400 pixel $\times 160$ pixel) proof set human terahertz image. 


\section{Mean Filtering Denoising THZ Images}

Before performing detection and identification, the human body terahertz image is denoised using a mean filter algorithm to facilitate subsequent processing. Its denoising effect is shown in Figure 2.

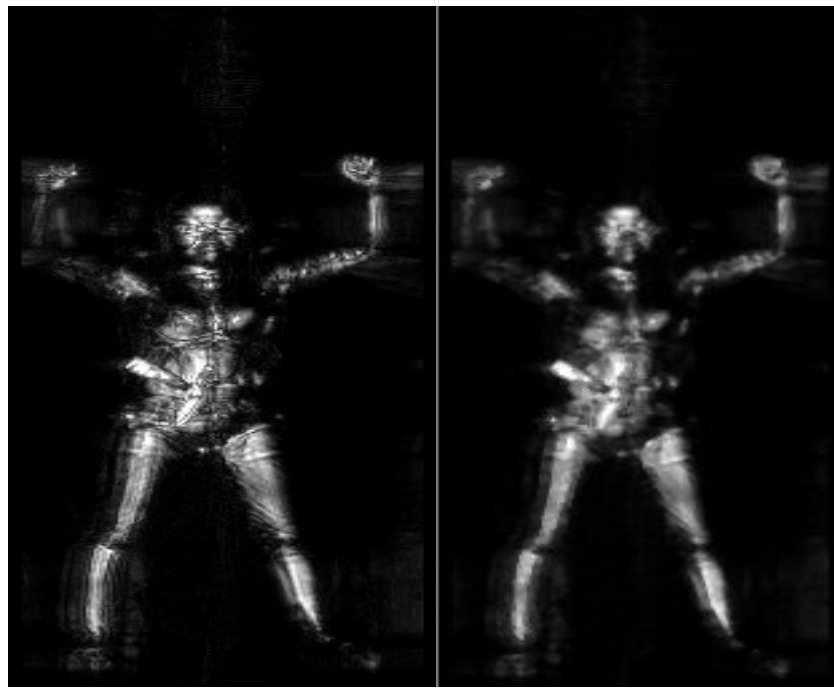

(a)Original image

(b)Denoising image

FIGURE 2. Human THZ images

\section{Faster RCNN Algorithm for Controlled Tool Recognition in Human THZ Images}

The detection and recognition of the human terahertz image after average filtering was performed, and the recognition rate parameter was adjusted to 0.8 . The test result was: 93 accurately labeled control tools, 14 misjudged and not recognized, and the recognition rate was $86.9 \%$. Some of these experiments are shown in Figure 3 :

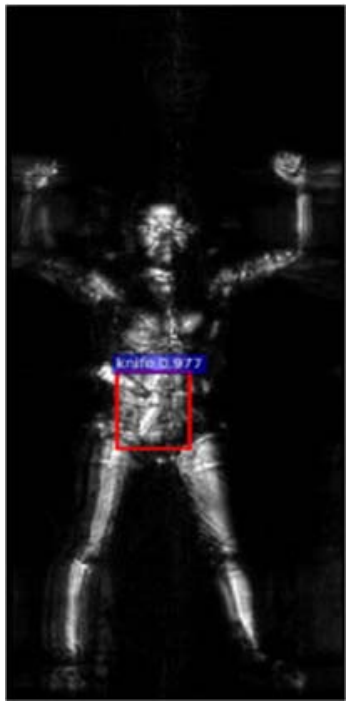

(a)

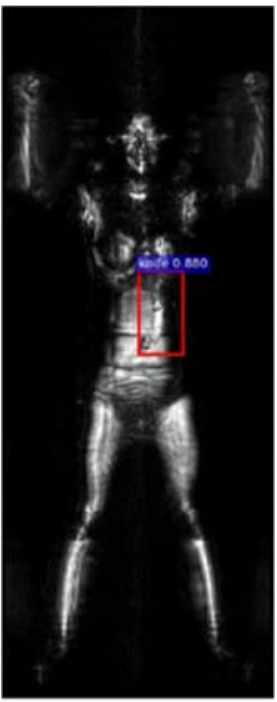

(b)

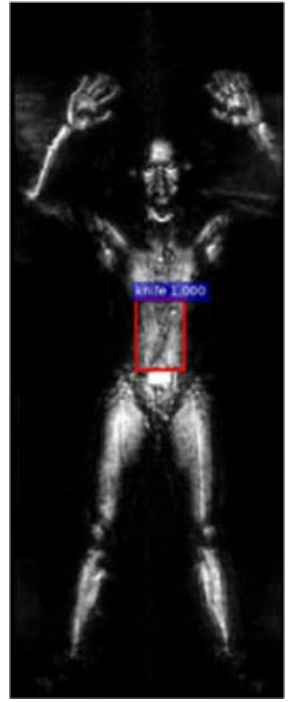

(c)

FIGURE 3. Experiment effect chart 


\section{Comparison with Other Algorithms}

The HOG-SVM algorithm, this algorithm and the YOLO algorithm were used to identify the controlled cutters in the human terahertz images, and the recognition rate and recognition speed were obtained. Experimental identification performance comparison as shown in Table 1

TABLE 1. Recognition accuracy and detection speed performance table

\begin{tabular}{cccc}
\hline & recognition rate & Time/CPU & Time/GPU \\
\hline HOG-SVM & $75.7 \%$ & 34 & -- \\
\hline Faster RCNN & $86.9 \%$ & -- & 2.7 \\
\hline YOLO & $84.1 \%$ & -- & 2.3 \\
\hline
\end{tabular}

From the experimental results, it can be seen that the Faster RCNN algorithm has faster recognition speed in better recognition rate. The use of HOG-SVM algorithm is relatively poor in recognition rate and recognition speed, the recognition rate is low, and the recognition speed is slow. The YOLO algorithm is fast, but its recognition rate is lower than that of the Faster RCNN. Therefore, the Faster RCNN algorithm is the most suitable for the identification of controlled tools in the human terahertz images.

\section{CONCLUSION}

After the human terahertz image is average filtered, the recognition rate of the controlled tool in the human terahertz images by the Faster RCNN is $86.9 \%$. Compared with the HOG-SVM algorithm and the YOLO algorithm, the overall performance is better, the recognition rate and the recognition speed. It is also more prominent. At present, the objects detected and identified in this paper have limitations. There are dangerous products with various types of narcotics, explosives, and even large differences in the actual screening, so the realization of multiple classifications meets the actual needs. In the future, more human terahertz images will be collected and there will be more dangerous species in the pictures, enriching the experimental data. The experiment not only recognizes human terahertz images containing knives and mobile phones, but also conducts experiments to identify and classify many types of dangerous goods. At the same time, it will also carry out enhanced research on terahertz images.

\section{REFERENCES}

1. ZHU Weiwen, ZHAO Yuejin, ZHU Huishi, LI Bing, KONG Lingqin, DONG Liquan. Application of improved mean filtering algorithm in terahertz imaging[J]. Infrared and Laser Engineering, 2013, 42(05): 1241-1246.

2. Sun Jianzhong, Zhang Le, Gao Fei, Li Jiusheng. Quality improvement and noise reduction of terahertz continuous wave imaging[J]. Spectroscopy and Spectral Analysis, 2017, 37(11): 3343-3346.

3. Feng Yanping, Wang Hui. Firearm THz image recognition based on threshold segmentation and edge detection [J]. Infrared, 2011, 32 (12): 23-26.

4. JIANG Tian, SHEN Huiliang, YANG Dongxiao, LIU Jianjun, ZOU Zhe. Terahertz image target detection based on C-means of fuzzy local information[J]. Laser Technology, 2015, 39(03): 289-294.

5. Shaoqing Ren, Kaiming He, Ross Grishick, Jian Sun, "Faster R-CNN: Towards Real-Time Object Detection with Region Proposal Networks", IEEE Transactions on Pattern Analysis and Machine Intelligence, pp. 11371149, 2016. 